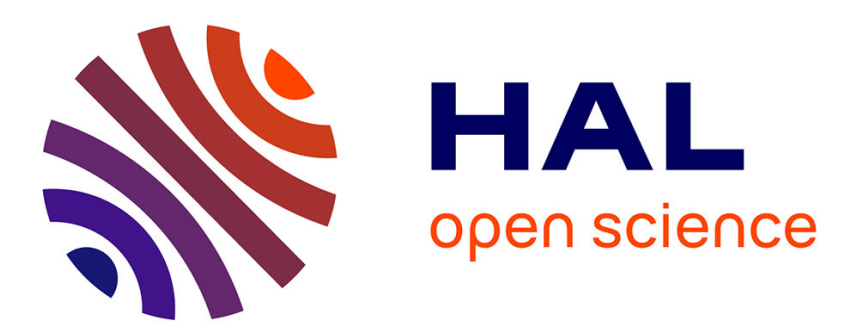

\title{
Distributed Contextualization of Biomedical Data: a case study in precision medicine
}

Sirine Sayadi, Estelle Geffard, Mario Südholt, Nicolas Vince, Pierre-Antoine

Gourraud

\section{- To cite this version:}

Sirine Sayadi, Estelle Geffard, Mario Südholt, Nicolas Vince, Pierre-Antoine Gourraud. Distributed Contextualization of Biomedical Data: a case study in precision medicine. AICCSA 2020 - 17th IEEE/ACS International Conference on Computer Systems and Applications, Nov 2020, Antalya, Turkey. pp.1-6, 10.1109/AICCSA50499.2020.9316502 . hal-02922930

\section{HAL Id: hal-02922930 \\ https://hal.inria.fr/hal-02922930}

Submitted on 26 Aug 2020

HAL is a multi-disciplinary open access archive for the deposit and dissemination of scientific research documents, whether they are published or not. The documents may come from teaching and research institutions in France or abroad, or from public or private research centers.
L'archive ouverte pluridisciplinaire HAL, est destinée au dépôt et à la diffusion de documents scientifiques de niveau recherche, publiés ou non, émanant des établissements d'enseignement et de recherche français ou étrangers, des laboratoires publics ou privés. 


\title{
Distributed Contextualization of Biomedical Data: a case study in precision medicine
}

\author{
Sirine Sayadi ${ }^{1,2}$, Estelle Geffard ${ }^{2}$, Mario Südholt ${ }^{1}$, Nicolas Vince ${ }^{2}$, Pierre-Antoine Gourraud ${ }^{2}$ \\ ${ }^{1}$ STACK team; IMT Atlantique, Inria, LS2N; Nantes, France; first. lasteimt-atlantique.fr \\ ${ }^{2}$ Centre de Recherche en Transplantation et Immunologie (CRTI); \\ CHU Nantes, INSERM, University of Nantes; Nantes, France; first.last@univ-nantes. fr
}

\begin{abstract}
An important aspect of precision medicine consists in patient-centered contextualization analyses that are used as part of biomedical interactive tools. Such analyses often harness data of large populations of patients from different research centers and can often benefit from a distributed implementation. However, performance and the security and privacy concerns of sharing sensitive biomedical data can become a major issue.

We have investigated these issues in the context of a kidney transplanted patient contextualization project: the Kidney Transplantation Application (KITAPP). In this paper, we present a motivation for distributed implementations in this context, notably for computing percentiles for contextualization. We present a corresponding system architecture, motivate privacy and performance issues, and present a novel distributed implementation that is evaluated in a realistic multi-site setting.

Index Terms - contextualization, precision medicine, kidney transplantation, distributed analysis, privacy, percentile
\end{abstract}

\section{INTRODUCTION}

Precision medicine strives for new biomedical methods and tools tailored towards the treatment of specific patients. Contextualization is then useful in order to evaluate medical data of individual patients with respect to larger groups of patients with similar characteristics. In particular, it can be used in order to compare patient markers relative to scales representing different severity levels. Clinicians can thus assess the condition of a patient and provide a perspective on her past, present and future state in order to help decide on a monitoring and a treatment [1].

As a recent example, consider the COVID-19 pandemic that has resulted in hundreds of thousands of lives lost and immense social and economic consequences. The dynamics of the spread of infectious diseases is often due to social behavior and puts enormous stress on existing social organizations and health infrastructures. Contextualization, modeling and understanding of statistical data and social behavior (panic, social distancing...) help decision-making on the best treatment in order to have a better response to the epidemic at (inter)national levels [2].

We have considered contextualization in the research project KITAPP (the Kidney Transplantation Application) [3], a precision medicine project for health-tracking of patients after kidney transplantations. This new tool aims at addressing the challenges of dynamic management of a complex chronic disease, notably the interaction of clinicians and patients in order to extend translational digital medicine and help personalized therapeutic decision. This project focuses on etiologically heterogeneous entities which can lead to different symptoms and treatments. It is therefore currently complicated, even for an experienced clinician, to predict a patient's clinical course and precisely anticipate the safety and efficacy of treatments. KITAPP relies on the contextualization of renal function outcomes (creatinemia, proteinuria...) of a patient of interest (POI) relative to a population of patients of reference (PORs). PORs are based on the identification of subgroups of patients with similar characteristics, such as age, gender and Body Mass Index (BMI).

Our contextualization algorithm consists of two steps. The first corresponds to the selection of a POR sub-population. As part of the second step we apply our modelization algorithm and visualize the contextualization of the POI w.r.t. the POR. This aspect of contextualization is performed using percentiles [4] as a major statistical tool that enables a patient to be positioned relative to other patients. A growth curve visualization system is proposed by modeling the individual trajectory of biological data (renal parameters routinely assessed in clinics) as a function of post-transplant follow-up, compared to the POR sub-population. Similarly, Corson et al. [5] provides a state-of-the-art example which uses percentiles to construct growth curves of pigs for use in biomedical studies.

The KITAPP project is a prototype laying the ground to different collaboration of clinical research currently recruiting patients, notably in two inter/national projects KTD-Innov [6] and EU-TRAIN [7]. So far, percentiles are calculated in a centralized way, only one site gathers all data.

Beyond technical issues, distributed architectures enable more flexible data governance strategies and processes by freeing them from centralization contraints [8]. Data management of health entities thus promises to be facilitated.

In order to deploy this kind of medical services in larger context, such as nation-wide or large international collaborations, distributed systems and algorithms for precision medicine have to be provided. Contextualization then has to be performed with respect to large-scale distributed medical databases that are maintained at different sites. Simple centralization schemes are not useful in this context because the necessary data may not be shared due to legal reasons, security/privacy concerns and performance issues. Moreover, 
approximate distributed calculations do not fit the requirements of precision medicine either.

In order to contextualize the state of a patient compared to a distributed database, fully-distributed analyses are thus very interesting, if they meet requirements of scalability, security and confidentiality [9]. Scheel et al. [10] discusses the importance of the availability, right to privacy and the accessibility of data in distributed biomedical research. These criteria are difficult to satisfy because the statistical significance and accuracy of analyses often directly depend on the number of cases or individuals included in the database.

In the context of distributed software systems and algorithms, data sharing and analysis is generally difficult due to governance, regulatory, scientific and technical reasons. Data protection policies, such as the EU General Data Protection Regulation (GDPR) impose well-founded, but strong restrictions on data sharing [11]. Hence, analyses are often only possible "on premise". Furthermore, researchers and institutions may be averse to lose control over both data and data usages. In addition, huge volumes of data are intrinsically difficult to share or transfer (e.g., cost associated to resource usage, such as computational, storage and network resources).

A solution to these problems can be found if a distributed analysis may be performed separately on the premises of the partners as far as sensitive data is concerned and if the global result of the analysis can then be calculated using aggregated, summarized or anonymized data. This principle is similar to but may require more complex algorithms than traditional divide-and-conquer algorithms that can often be distributed in terms of master-worker systems.

In this paper, we present two main contributions:

- We motivate and define requirements for distributed algorithms for contextualization in the context of the KITAPP project.

- We present a novel distributed percentile algorithm for contextualization in the presence of sensitive data in precision medicine.

The rest of this article is organized as follows: Section II presents the Kidney transplantation application (KITAPP), contextualization algorithms and the need for distributed analyses of these algorithms for precision medicine. Section III presents our distributed algorithm, a corresponding implementation and results of a detailed experimentation. Finally, Section IV summarizes our findings and proposes some future work.

\section{THE KIDNEY TRANSPLANTATION APPLICATION}

(KITAPP)

Chronic kidney failure affects approximately $10 \%$ of the world population and can progressively lead to end-stage kidney disease requiring replacement therapy (dialysis or transplantation). Kidney transplantation is the best treatment for end-stage kidney disease [12]. The one-year survival rate of kidney transplant is now $90 \%$ and the graft half-life is about 10 years. Patient follow-up is critical to monitor graft and patient's health; clinicians have several ways of monitoring kidney function, in particular creatinemia: creatinine level is measured to assess kidney filtration rate. Modern tools such as KITAPP is a new addition to clinicians way of performing optimal care.

\section{A. KITAPP Overview}

Data from approximately 1500 renal transplantation, including clinical and immunological items, were collected since 2008 as part of the DIVAT cohort.

KITAPP enables personalized contextualization algorithm to be harnessed to compare data trajectories of a given patient (POI) to a sub-population with similar characteristics (POR) selected by filters or distance measures. The information relative to a graft is selected from similar cases at the time of the graft. With the help of clinicians and knowledge of the existing body of research, we defined a set of variables to select the sub-population of reference. The reference sub-population has been defined either by filtering w.r.t. one or several of these variables or by applying a statistical strategy. A statistical strategy performs selection based on a statistical size reduction technique that reduces data from a large dimensional space to data in a smaller dimensional space. In our case, a reference population has been determined based on different statistical methods: nearest neighbor approach (select individuals more similar to a POI) and a clustering approach (select individuals in the same cluster as a POI).

The visualization of contextualized information is done by comparing a POI biological data (creatinemia) and its evolution over time (clinical visits) to a POR that is represented by their median and percentile values.

\section{B. Motivation for Distributed Analyses for KITAPP}

Data exchange requirements for medical multi-center/multipartner collaborations have led to a paradigm shift in medical data sharing system. Traditionally, centralized infrastructures have been used for storing, processing or archiving information. Since the enactment of the GDPR, these structures are often no longer suitable for collaborative health projects due to restrictions on access to sensitive data. To meet the challenge of harnessing medical data while keeping sensitive data on premise or ensure strong data protection if data is moved, computations are often performed today over distributed databases that are linked to a computation integrator that enables a center to interact with and access some data from remote sites. Each clinical center collects, stores and controls their own patients' data. The founding principle of the architecture is that no data of individuals circulates outside the centers. However, this sharing paradigm is very restrictive and inhibits a large range of potential analyses to be performed - either because sensitive data cannot be appropriately protected or the analysis cannot be performed sufficiently efficient. We are working on more general distributed analysis architectures and implementations that ease collaboration as part of multi-centric research projects, where each center can control and account for their own patients' data usage even if located remotely. 


\section{Contextualization of Distributed Biomedical DATA}

In the context of the KITAPP project, we have worked on the distributed contextualization of parameters of renal activity. In the following we present a distributed algorithm for computing percentile information over data distributed among different sites that shares only small amounts of data and no sensitive data between the sites.

\section{A. Algorithmic principle}

The rank of a percentile is defined as follows:

$$
k=P / 100 * N
$$

where $N$ is the number of values in the data set, $P$ the percentile and $k$ the rank of a given value. The calculation of the value defining a percentile thus requires to first compute $k$ and then identify the corresponding value. Identifying the $k^{\text {th }}$ value is difficult and costly in an unsorted array but simple and efficient in an ordered one. Furthermore, gathering all relevant elements necessary to determine a given percentile or first ordering the available values over all sites may result in a large proportion of all values to be moved among sites. Both types of such simple approaches may thus suffer serious issues from the efficiency and data protection points of view.

We therefore have investigated algorithms that require few exchanges and sharing of data. We build on the well-known (non-distributed) QuickSelect algorithm (developed by Hoare in 1961 [13]) that permits to select the $k^{\text {th }}$ largest element in an unsorted array by partially ordering that array. Similar to QuickSort, QuickSelect partitions an array according to a pivot element that may be arbitrarily chosen; in contrast to the sorting algorithm, QuickSelect recursively partially orders and searches only one of the partitions: the partition which contains the target value. QuickSelect achieves an average complexity of $O(n)$ (and $O\left(n^{2}\right)$ in the worst case of an unlucky choice of the pivot element).

\section{B. Distributed implementation}

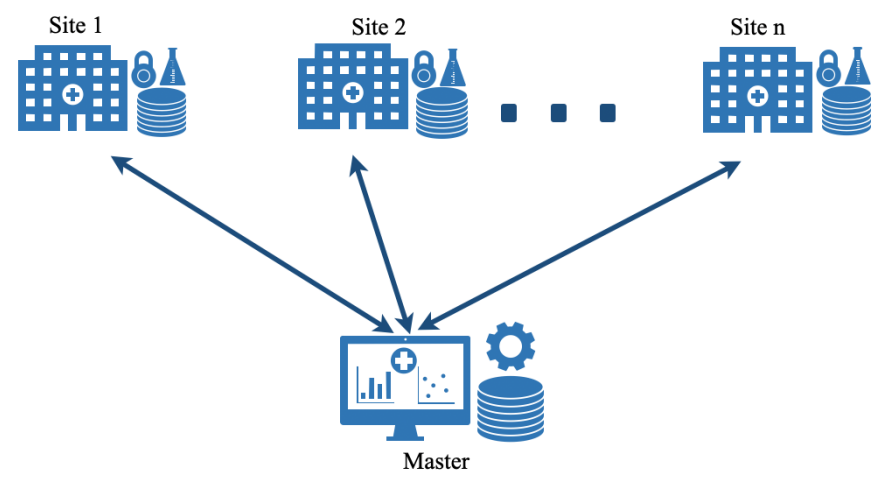

Fig. 1. Collaboration Requirements.

As part of our medical cooperation we are interested in a collaboration structure as illustrated in Fig. 1. The overall analysis is performed on multiple sites, each of which is operating under coordination of a master site. However, in contrast to many fully data-parallel master-worker schemes our analysis has to observe dependencies among the worker nodes. Saukas and Song [14] have proposed a parallel algorithm for the selection of the smallest element in a set of elements. This algorithm requires few communication and also shares few data. We have used the same algorithmic principle and adapted it to large-scale distributed environments, notably by implementing data exchanges and sharing.

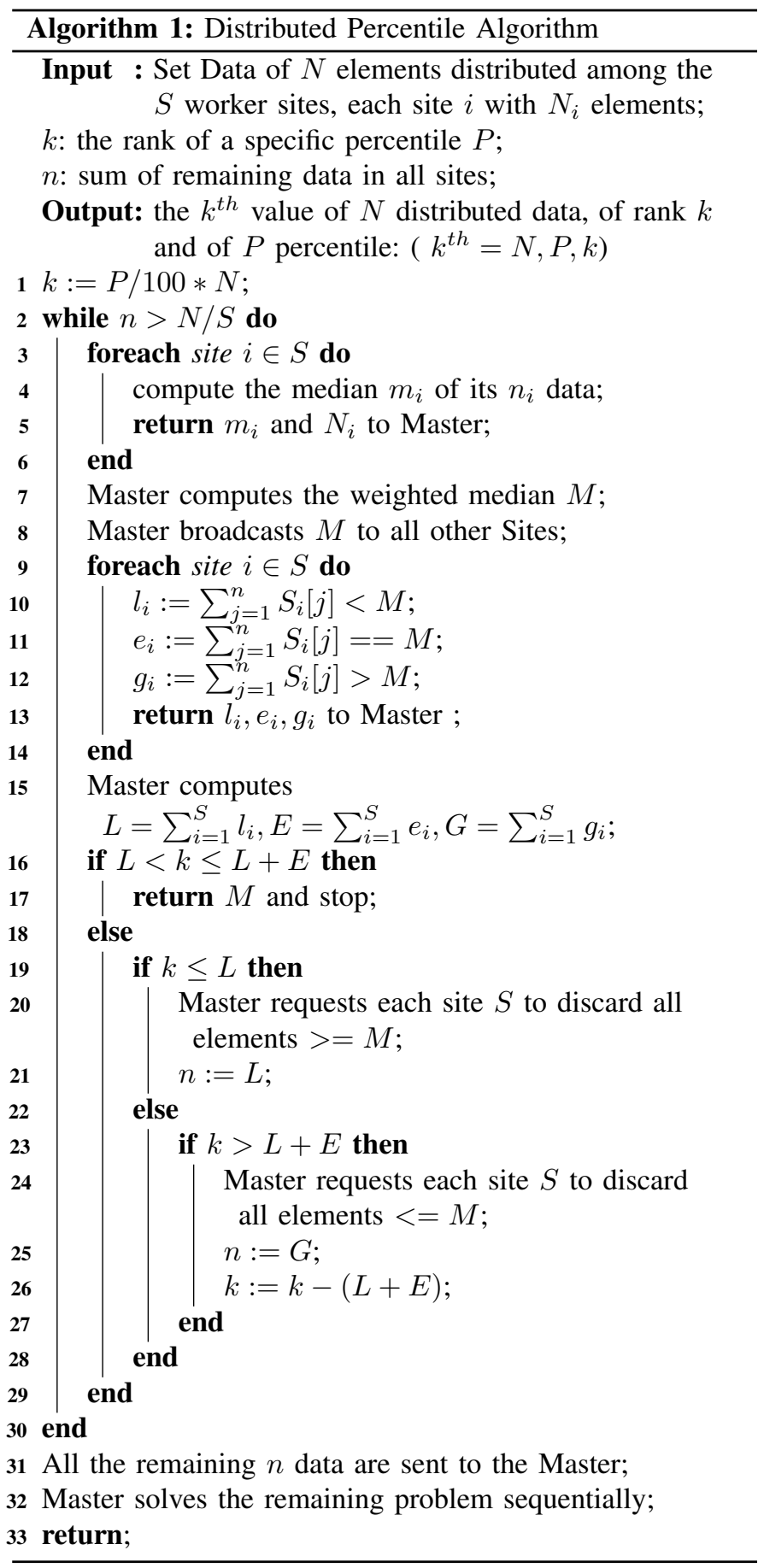

The pseudocode of our distributed algorithm is shown in 
Algorithm 1. The master first calculates the rank $k$ of the percentile relative to $N$, the total size of the data over all hospitals. Each worker starts by sending the size of their data $N_{i}$ as well as the local median $m_{i}$. Then the master calculates the weighted median $M$ and broadcasts $M$ to all workers. Each worker then calculates $l_{i}, e_{i}, g_{i}$ which correspond, respectively, to the number of values smaller, equal and greater to $M$ and returns them to the server, after which the server calculates the respective sums $L, E$ and $G$. If $(L<k<=L+E)$ then the result corresponds to $E$. If $(k<=L)$ then the master asks each worker to discard all the values smaller than $E, n$ is set to $L$. If $(k>L+E)$ then the master asks each worker to discard all the values greater than $M$ and $n$ is set to $G, k$ to $k-(L+E)$. This process is repeated until $(n<=N / S)$. Finally, if we do not arrive at the solution after this parallel analysis, the server begins the same analysis sequentially on the rest of the data $n$.

\section{Experimentation}

We have implemented our algorithm and a driver program to deploy and executed it in a grid-based environment whose nodes may consist of individual machines but also fullfledged clusters. Note that this environment closely mimics the architecture of real medical cooperations whose different partner sites form a grid and individual partners may dispose of computational clusters themselves. The distributed algorithm has been implemented using Python consisting of 840 LoC; its deployment and execution using an, in principle, arbitrary number of worker nodes just requires eight commands on the Grid'5000 infrastructure. We have tested our algorithm extensively and successfully by comparing its result to its sequential counterpart.

We have applied the percentile contextualization analysis to real validated transplantation data available from the French DIVAT database [15]. In order to support the KITAPP project, we have explored the (distributed) contextualization of creatinine levels in patients. So we took creatD as a tracking variable for contextualization of the creatinine level in patients. After extracting relevant data for this analysis, we obtained a file of 11,028 values. We divided this file into several files of different sizes for distribution over different numbers of worker sites.

\section{Geo-distributed infrastructure deployment}

To carry out our experimentation, we used the Grid'5000 platform [16] as a real-world grid and cluster-based experimentation environment. Grid'5000 is an infrastructure dedicated to CS research in the fields of large-scale distributed systems, high performance computing and networking. For each experiment, we have reserved machines which are used as workers as shown in Fig. 2 located in five different French cities. These machines contain the data stored as CSV files as well as the worker code. We have also reserved a machine as master (server in computational terms) which will perform the analysis by communicating with the workers using socket communication without having access to the data of the CSV

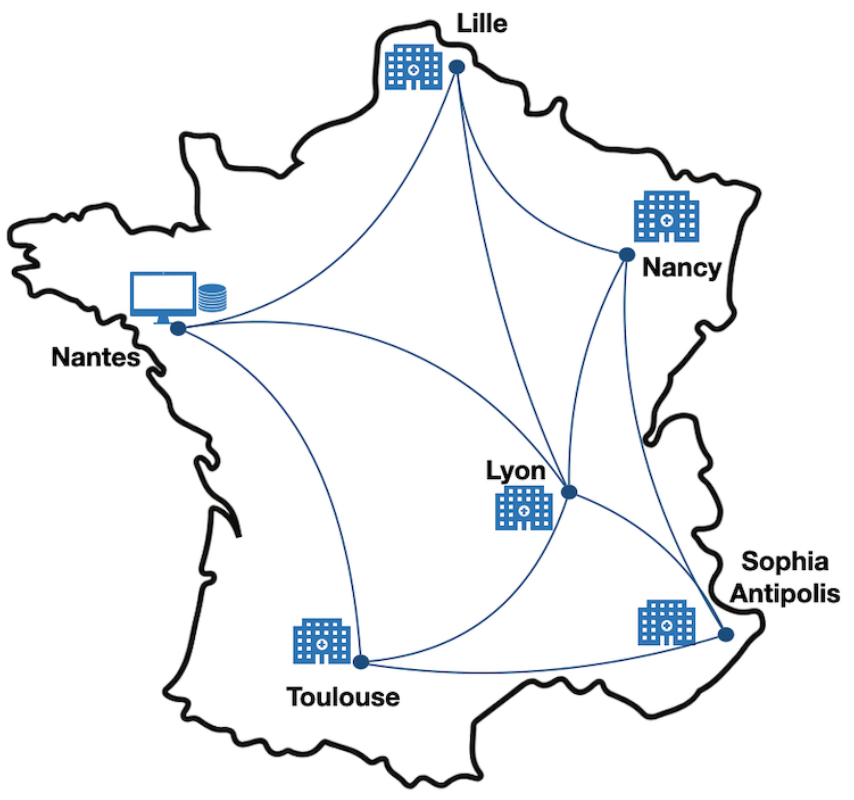

Fig. 2. Experimentation with Grid'5000 testbed.

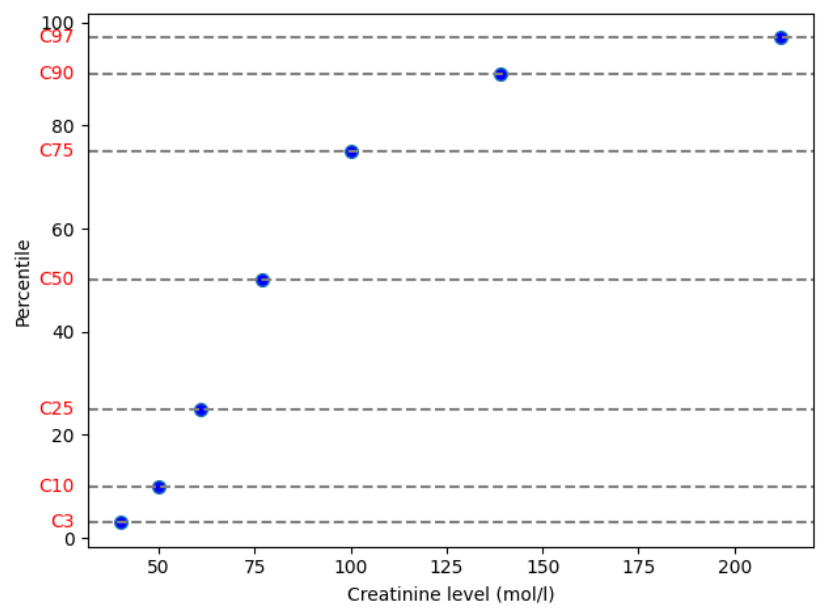

Fig. 3. Contextualization of creatinine level (mol/l).

files and generating the percentile result at the end of analysis, thus enabling the visualization of the creatinine levels of a given patient compared to the others.

Fig. 3 shows our results for creatinine level contextualization of patients computed on five worker sites. This figure shows the state of creatinine level in patients compared to others based on five percentile results $\left(3^{r d}, 10^{t h}, 25^{t h}, 50^{t h}\right.$ and $97^{t h}$ percentile). For example, the $25^{t h}$ and the $50^{t h}$ percentile correspond to people who have a creatinine level of respectively $61(\mathrm{~mol} / \mathrm{l})$ and $77(\mathrm{~mol} / \mathrm{l})$ compared to the population distributed over the five sites.

\section{E. Evaluation}

We have evaluated our system with respect to two types of parameters: performance-related parameters (different number of sites, different data sizes, execution time) and parameters 


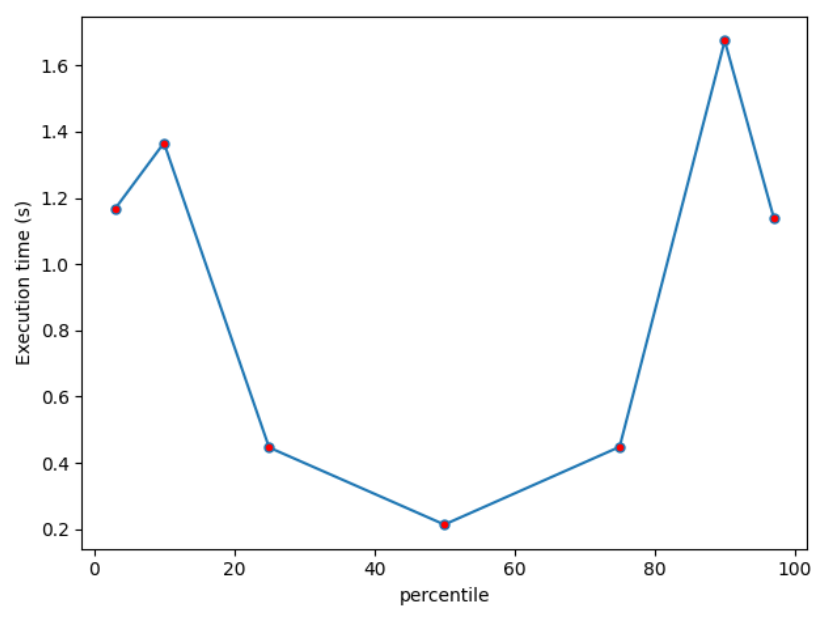

Fig. 4. Execution time for 5 sites.

related to data sharing (proportion of shared data by percentile, data size and number of sites).

Execution time by percentile. We have measured the execution time for each percentile performed. Fig. 4 presents the time to complete each percentile for the case of 5 sites. the $x$ axis shows the calculated percentiles and the y-axis shows the time needed to calculate each percentile. The $50^{\text {th }}$ percentile takes slightly less time compared to the other percentiles $(0.2 \mathrm{~s}$ less) because we can sometimes dispense with the sequential phase of the algorithm in this case. This shows the interest of distributed analyses not only for reasons of confidentiality and privacy but also for reasons of performance.

Scalability by number of sites. We have also evaluated the execution time of our proposed approach with respect to different numbers of worker sites by partitioning a fixed data set on $3,5,7,10$ and 15 sites. Fig. 5 presents the execution time of $3^{r d}, 10^{t h}, 25^{t h}, 50^{t h}, 75^{t h}$ and $90^{t h}$ percentiles for data partitioned on $3,5,7,10$ and 15 sites. The figure shows that the percentile calculation time increases with the increase in the number of sites but it always remains very small and does not exceed $3.2 s$ for the calculation of $90^{t h}$ percentile on 15 sites for a data set of size 11028 .

Scalability by data size. We have also evaluated the efficiency of our proposed algorithm on a large number of data and a large size of data compared to the previously mentioned set. Table I shows the number of data and the data sizes used in these experiments.

TABLE I

NUMBER OF DATA FOR EACH SIZE

\begin{tabular}{|l|l|}
\hline Size of global data (MB) & Number of global data points \\
\hline 0.131 & 11028 \\
\hline 0.363 & 110280 \\
\hline 3.6 & 1102800 \\
\hline 36.3 & 11028000 \\
\hline 363 & 110280000 \\
\hline
\end{tabular}

Fig. 6 shows the execution time of our approach to calculate

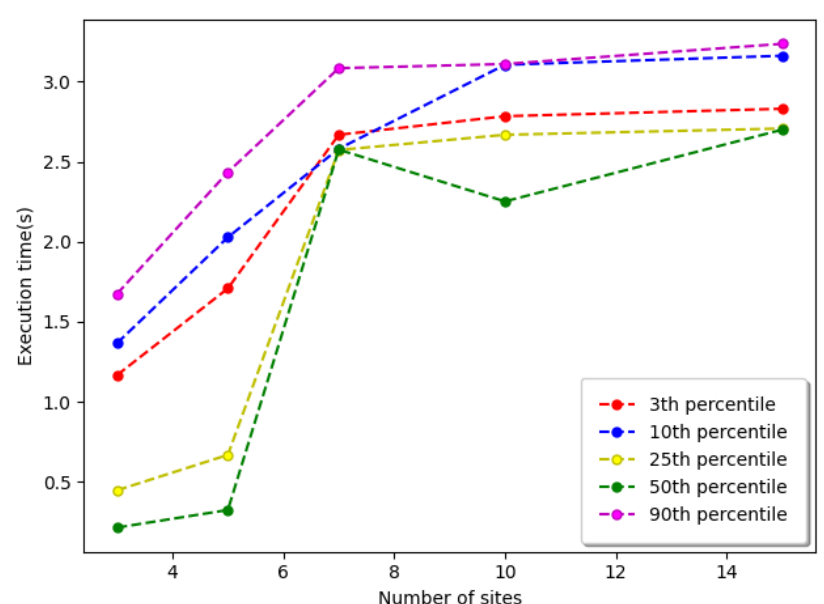

Fig. 5. Execution time by clients number.

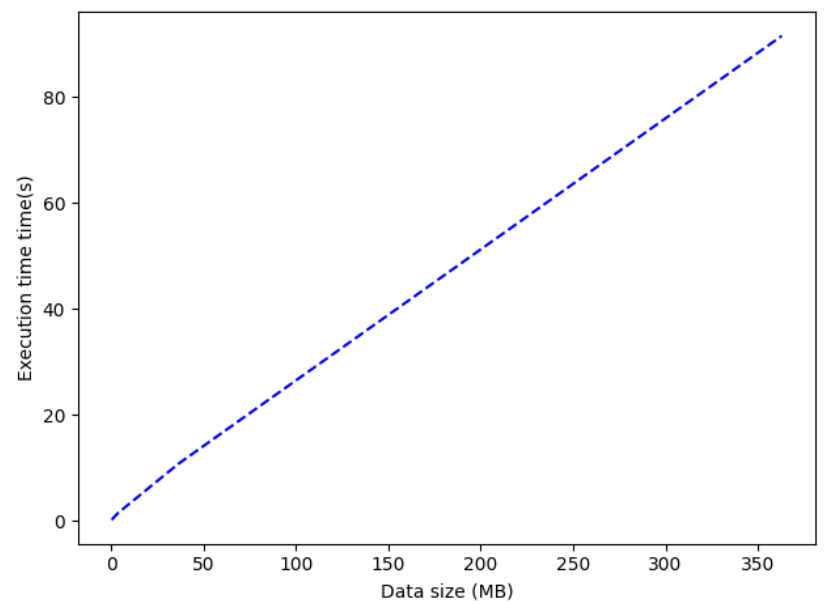

Fig. 6. Execution time per $50^{\text {th }}$ percentile by data size.

the $50^{\text {th }}$ percentile for the case of 3 sites with different sizes of global data. We can notice that the execution time of our algorithm increases linearly in the data size while yielding exact results compared to a centralized calculation.

Privacy by percentage of shared data. Another important parameter in the medical context is the amount of data shared, in particular as part of the sequential phase of our algorithm. We carried out two experiments in order to assess the percentage of data shared by increasing the overall size of the data or the number of sites participating in the analysis.

The results are presented in Figures 7 and 8 in form of boxand-whisker diagrams that provide a detailed vision on the distribution of shared data, making explicit medians, lower and upper quartiles (as boxes) and outliers (as green stars). The figures show that the percentage of shared data never exceeds $0.89 \%$ compared to all the data for all the sites, which is negligible especially because shared data only consists of aggregate values (medians) that do not match any sensitive data that can identify a patient. The median of the proportion 


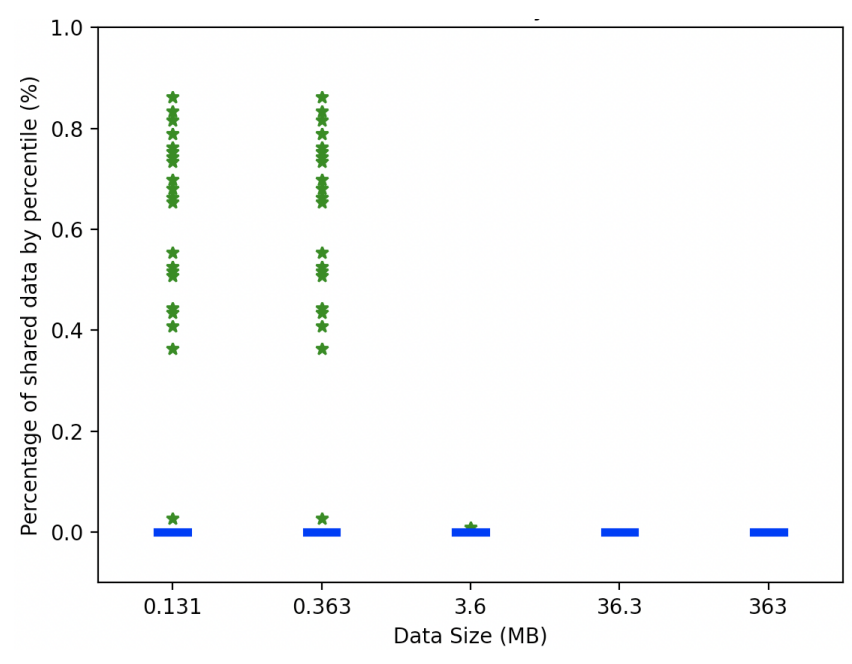

Fig. 7. Percentage of shared data by data size.

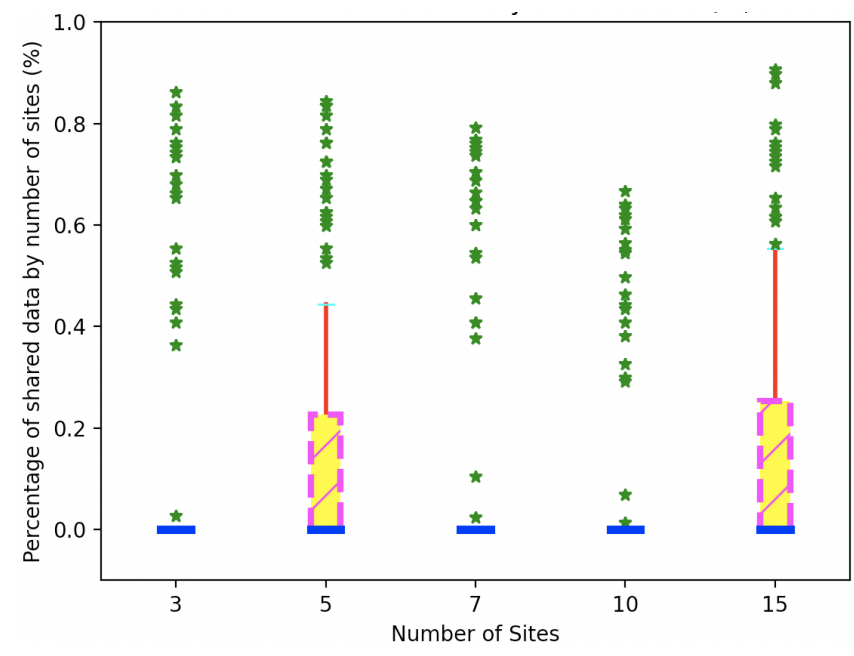

Fig. 8. Percentage of shared data by number of sites.

of shared data is close to 0 , which explains that in most cases the algorithm only performs parallel computations without requiring recourse to sequential solving steps and thus without any sharing of the data. The two figures show some outliers between $0.3 \%$ and $0.89 \%$ especially in all cases when the number of sites participating in the analysis is increased. Furthermore, Fig. 7 shows that, if we increase the size of the data, our algorithm will be even more efficient in terms of percentage of shared data which is decreasing until it is almost 0 .

The accuracy of our algorithm is preserved even if we increase the number of distributed sites and the size of data while keeping the confidentiality of the data with the distributed model by keeping a percentage of the shared data which does not exceed $0.89 \%$ compared to global data.

\section{CONCLUSION AND FUTURE WORK}

Contextualization is an important tool that allows clinicians and medical experts to assess the state of data and help decision-making with an interpretation of statistical analyses of the data. In order to respect the confidentiality of data and perform this type of analysis on a large scale, we have proposed in this paper a novel distributed contextualization algorithm for percentiles that requires only few sharing of data between sites. We have motivated our approach in the context of real-world contextualization in the domain of medical transplantations. We have carried out realistic experiments over real data in a realistic grid-cluster based distributed environment. Our experiments have shown accurate results compared to a centralized contextualization analysis with very good performance results and scalability in terms of system size and execution time. Finally, we have shown that our algorithm requires only very little data sharing that does not involve sharing of individual sensitive data.

As future work in distributed contextualization, we will focus on distributed factor analysis of incomplete data with mixed quantitative and qualitative variables from different sources.

\section{ACKNOWLEDGMENT}

This work is supported by KTD-innov Project. The experiments presented in this paper were carried out using the Grid5000 experimental testbed, see www.grid5000.fr. It has also been supported by the EU's Marie Skłodowska-Curie grant agreement no. 846520 .

\section{REFERENCES}

[1] P. Gourraud, R. Henry et al.: "Precision medicine in chronic disease management: the MS BioScreen," Ann Neurol, 76(5): 633-642, 2014.

[2] A. Jarynowski, M. Kempa et al.: "Attempt to Understand Public Health Relevant Social Dimensions of COVID-19 Outbreak in Poland,' Society Register, 4(3): 7-44, April 2020.

[3] C. Herve, N. Vince et al.: "P218 The kidney transplantation application (KITAPP): A visualization and contextualization tool in a kidney graft patients' cohort," Human Immunology, Volume 78, Page 216, 2017.

[4] R. Hyndman and Y. Fan, "Sample quantiles in statistical packages," The American Statistician. 50 (4): 361-365, 1996.

[5] AM. Corson AM, J. Laws et al.," Percentile growth charts for biomedical studies using a porcine model,”. Animal : an International Journal of Animal Bioscience, 2(12):1795-1801, 2008.

[6] KTD-Innov. www. ktdinnov. fr (visited on 15/06/2020).

[7] EU-TRAIN. eu-train-project.eu .

[8] P. Brous, M. Janssen et al.: "Coordinating Decision-Making in Data Management Activities: A Systematic Review of Data Governance Principles,' Int. Conf. on Electronic Government, 2016, Springer.

[9] F. Boujdad, A. Gaignard et al.: "On Distributed Collaboration for Biomedical Analyses"; WS CCGrid-Life, 2019.

[10] H. Scheel , H. Dathe, T. Franke, T. Scharfe, T. Rottmann, "A Privacy Preserving Approach to Feasibility Analyses on Distributed Data Sources in Biomedical Research," Studies in Health Technology and Informatics, 267:254-261, 2019.

[11] GDPR. https://eur-lex.europa.eu/legal-content/FR/ TXT/HTML/ ? uri=CELEX: 32016R0 679 (visited on 13/06/2020).

[12] N. Hill, S. Fatoba et al: "Global Prevalence of Chronic Kidney Disease - A Systematic Review and Meta-Analysis," PLoS One. 2016.

[13] Hoare, "C. A. R. : Algorithm 65: Find," Comm. ACM. 4 (7): 321-322, 1961.

[14] E.L.G. Saukas, S. W. Song: "A Note on Parallel Selection on CoarseGrained Multicomputers"; Algorithmica 24: 371-380, 1999.

[15] Divatfrance. www. divat. fr (visited on 15/06/2020).

[16] D. Balouek, A. Carpen Amarie, G. Charrier et al.: "Adding virtualization capabilities to the Grid'5000 testbed," in Cloud Computing and Services Science. Springer, 2013. 